\title{
Differential Gene Expression in Cell Types of the Human Skeletal Muscle: A Bioinformatics-Based Meta-Review
}

\author{
Kyung-Wan Baek ${ }^{1,3}$ PhD, So-Jeong Kim ${ }^{2}$ BS, Ji-Seok Kim ${ }^{1,3}$ PhD, Sun-Ok Kwon ${ }^{1}$ PhD \\ ${ }^{1}$ Department of Physical Education, Gyeongsang National University, Jinju; '²Department of Convergence Medical Science, Gyeongsang National University, Jinju; \\ ${ }^{3}$ Research Institute of Pharmaceutical Sciences, Gyeongsang National University, Jinju, Korea
}

PURPOSE: This study evaluates the differences in the expression of genes frequently analyzed in the field of exercise science between the skeletal muscle tissue and various cell types that comprise the skeletal muscle tissue.

METHODS:We summarized the genes and proteins expressed in the skeletal muscle that were published in "Exercise Science" journal from 2015 to present. Thereafter, we selected 15 genes and proteins that were the most analyzed genes and proteins in the skeletal muscle. These genes and proteins were horizontally compared for expression differences in skeletal muscle components and cultured cells based on NCBI Gene Expression Omnibus DataSets.

RESULTS: The most analyzed genes (encoding analyzed proteins) in skeletal muscle tissues in "Exercise Science" were PPARGC1A, PPARD, MTOR, MAP1LC3A, MAP1LC3B, PRKAA1, AKT1, SLC2A4, MAPK1, COX4I1, MAPK14, MEF2A, MAPK8, RPS6KB1, and SOD1. Among them, PPARGC1A, AKT1, SLC2A4, MAPK1, and COX4I1 were specifically expressed in the skeletal muscle. However, expression of other genes was found to be significantly affected in other cell types of the skeletal muscle tissue.

CONCLUSIONS: Genes such as PPARGC1A, which are specifically expressed in the skeletal muscle, may be analyzed without pretreating (such as perfusion) the skeletal muscle tissue. However, expression of other genes may depend on the skeletal muscle cell type. Thus, in such instances, pretreatment, such as perfusion and isolation, should be considered.

Key words: Skeletal muscle, Gene, Gene expression, Bioinformatics, Exercise

\section{INTRODUCTION}

One of the main areas of interests in exercise science is investigating the role of exercise in regulating gene expression in the skeletal muscle, as it causes physiological changes to the skeletal muscle [1,2]. Therefore, it is important to accurately analyze gene expression in the skeletal muscle.

A tissue sample, obtained via a muscle biopsy that has not been subjected to an isolation process has different proportions of the formative cells $[3,4]$. This is because skeletal muscle mainly consists of muscle fibers; however, it also contains blood and lymph vessels, nerves, and immune cells. Each of these cell types can potentially respond to experimental conditions; therefore, it is difficult to decipher the exact role of muscle cells in the overall tissue response. For example, the surface marker CD14 is expressed on circulating monocytes [5]. Thus, the mRNA expression of CD14 is higher in blood cells and primary macrophages than in other cell types [6]. In addition, it was demonstrated that endothelin expression is specific to endothelial cells and is much higher in these cells than in muscle or blood cells [6]. Conversely, the mRNA levels of myosin heavy chain 2 are substantially high in biopsied muscle tissues, fibers, and cells; however, it is rarely expressed in blood, immune, and endothelial cells [7].

Since the skeletal muscle is a mixture of different cell types, it is necessary to pretreat the biopsied muscle or develop a statistically accurate technique such that the expression of the gene of interest can be deter- 
mined. Moreover, it is experimentally challenging to evaluate the differences in gene expression in various cell types. Nevertheless, prior to experimentally validating the differential expression of genes in the skeletal muscle, a quick preliminary analysis and prediction can be conducted using the genome database available in NCBI Gene Expression Omnibus (GEO) DataSets (https://www.ncbi.nlm.nih.gov/gds/). An open access genome analysis tool that determines the baseline expression levels of a gene has become recently accessible. Recently accessible open access gene expression analysis tool maintain and process only baseline/control conditions for each microarray uploaded in NCBI GEO DataSets [8].

In this paper, we discuss a rational approach to interpret the expression of genes and proteins most analyzed in the skeletal muscles in the journal "Exercise Science" using a genome dataset-based online analysis tool.

\section{METHODS}

\section{Identifying the most analyzed genes and proteins in "Exercise Science"}

We reviewed all the papers published in the journal "Exercise Science" from 2015 to present and identified all the genes and proteins analyzed in the skeletal muscle. Thereafter, we matched the identified genes to the species used in the experiment of each published papers by accessing the NCBI Gene database (https:/www.ncbi.nlm.nih.gov/gene/). Similarly, we cross-referenced the identified proteins with the NCBI Gene database and identified the corresponding coding genes (Table 1). Among the identified genes, those that were homologous among species were considered as identical genes. All genes identified through the process described above were converted from mouse (Mus musculus) or rat (Rattus norvegicus) to human (Homo sapiens) gene symbols. Eventually, we ranked these analyzed genes, as shown in Table 2. Each publications within the "Exercise Science" journal included in this study contained one or more of the keywords "skeletal muscle", "muscle", "gene", "gene expression" and "expression".

\section{Differential gene expression in cell types of the skeletal muscle}

Expression levels of the converted genes were analyzed using the RNA expression analysis tool (https://nicopillon.com/tools/muscle-atlas/) based on the NCBI GEO DataSets. Subsequently, we compared the expression levels of genes in the muscle fibers, whole blood cells, endotheli- al cells, fibroblasts, macrophages, muscle cells, and smooth muscle cells with those of genes expressed in tissues obtained from muscle biopsy. All of the analyzed genes were mentioned more than twice in the "Exercise Science".

\section{Statistical analysis}

After calculating the relative gene expression values $\left(\log _{2}\right)$ based on cellular characteristics, we performed one-way ANOVA and Dunnett's multiple comparisons tests using GraphPad Prism (version 8.3.0, GraphPad Software, Inc., La Jolla, CA, USA). Statistical significance was set at $p<.05$.

\section{RESULTS}

\section{Publications reporting gene and protein expression in skeletal muscle cells were analyzed in the "Exercise Science" journal from 2015 to present}

Upon reviewing publications in the "Exercise Science" from 2015 to the present, we determined that 18 studies were interested in skeletal muscle genes or proteins. Of these, nine studies were conducted on mice, and seven were conducted on rats. In addition, one study was conducted in both mice and rats. Furthermore, one of the remaining studies was a genome meta-analysis. We found that the most analyzed skeletal muscle was the tibialis anterior, which was analyzed in six studies. In addition, the soleus, gastrocnemius, triceps, extensor digitorum longus, and flexor hallucis longus were the most frequently analyzed muscles in this order (Table 1).

\section{Most analyzed genes and proteins in the "Exercise Science" journal from 2015 to the present}

The most analyzed gene (or encoded protein) in the journal was PPARGC1A (encoding PPARG co-activator 1 alpha $[\mathrm{PPAR} \beta / \delta]$ ), which was identified in five publications. The next most analyzed gene was PPARD (encoding peroxisome proliferator activated receptor delta $[\operatorname{PPAR} \beta / \delta]$ ), which was analyzed in three publications. In addition, MTOR (encoding mechanistic target of rapamycin [mTOR]), MAP1LC3A (encoding microtubule associated protein 1 light chain 3 alpha [LC3]), MAP1LC3B (encoding microtubule associated protein 1 light chain 3 beta [LC3B]), PRKAA1 (encoding protein kinase AMP-activated catalytic subunit alpha 1 [AMPK]), AKT1 (encoding AKT serine/threonine kinase $1[\mathrm{AKT}]$ ), SLC2A4 (encoding solute carrier family 2 member 


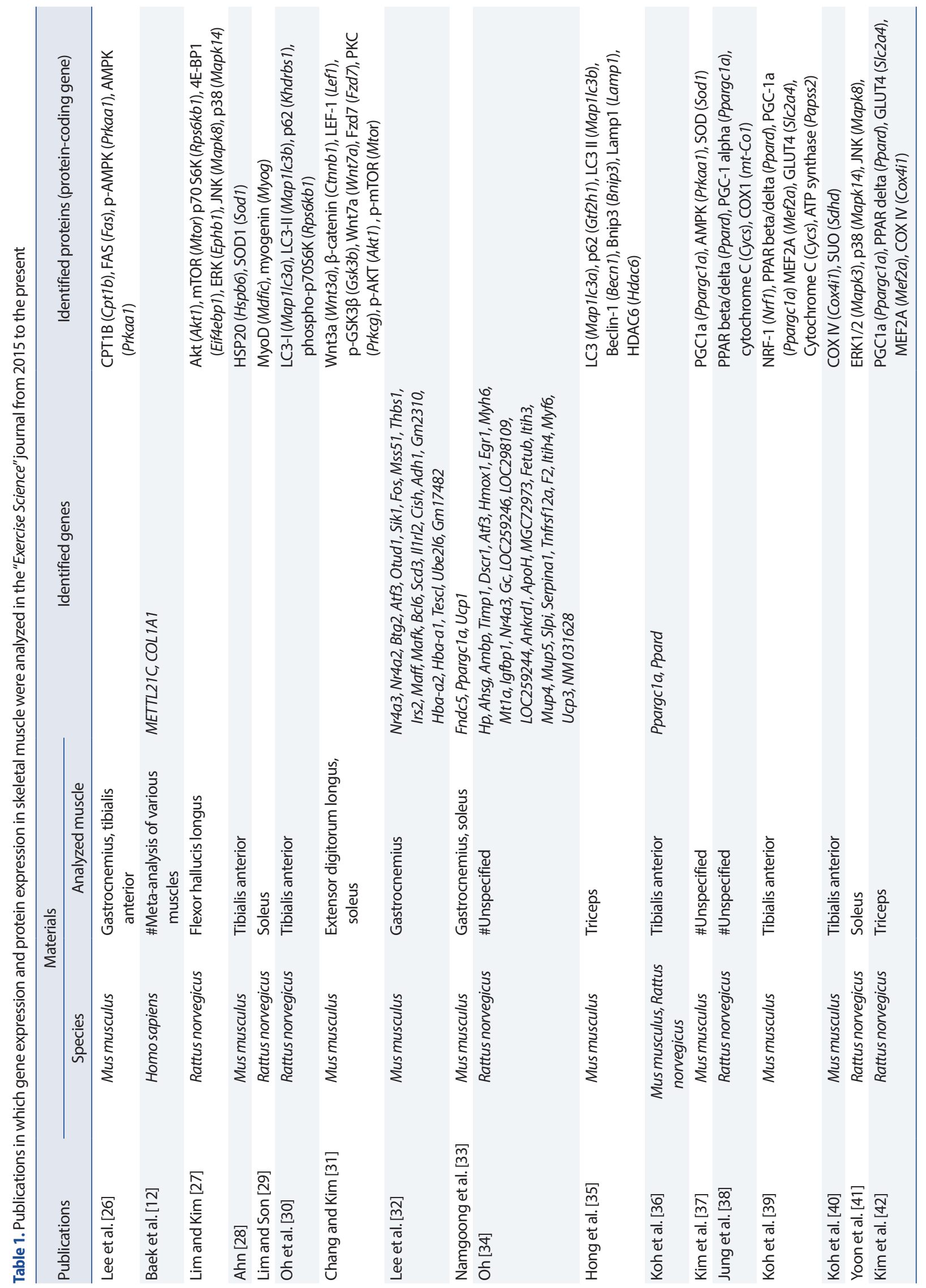


Table 2. Most analyzed genes in skeletal muscle in published "Exercise Science" journals

\begin{tabular}{lcc}
\hline Rank & Gene symbol of Homo sapiens & Number of publications \\
\hline 1 & PPARGC1A & 5 \\
2 & PPARD & 3 \\
3 & MTOR & 2 \\
3 & $M A P 1 L C 3 A$ & 2 \\
3 & $M A P 1 L C 3 B$ & 2 \\
3 & $P R K A A 1$ & 2 \\
3 & AKT1 & 2 \\
3 & SLC2A4 & 2 \\
3 & $M A P K 1$ & 2 \\
3 & COX4I1 & 2 \\
3 & $M A P K 14$ & 2 \\
3 & $M E F 2 A$ & 2 \\
3 & $M A P K 8$ & 2 \\
3 & $R P S 6 K B 1$ & 2 \\
3 & $S O D 1$ & 2 \\
\hline
\end{tabular}

4 [GLUT4]), MAPK1 (encoding mitogen-activated protein kinase 1 [ERK]), COX4I1 (encoding cytochrome c oxidase 4I1 [COX4]), MAPK14 (encoding mitogen-activated protein kinase 14 [p38]), MEF2A (encoding myocyte enhance factor 2A [MEF2]), MAPK8 (encoding mitogen-activated protein kinase 8 [JNK]), RPS6KB1 (encoding ribosomal protein S6 kinase B1 [p70-S6K]), and SOD1 (encoding superoxide dismutase 1 [SOD]) were analyzed in two publications each.

\section{Cell-type based differential expression of the most studied genes in "Exercise Science"}

We found that the expression of PPARGC1A did not differ substantially between muscle-biopsy obtained tissue samples and muscle fibers. However, the expression in PPARGCLA significantly differed in all other cell types ( $p<.0001$; Fig. 1A). On the other hand, PPARD expression differed insignificantly between muscle-biopsy obtained tissue samples, muscle fibers, endothelial cells, and muscle cells. Nevertheless, we validated that PPARD expression significantly differed in whole blood cells $(p<.0001)$, fibroblasts $(p<.0001)$, macrophages $(p<.0001)$, and smooth muscle cells $(p<.001)$ compared to that in biopsied tissue samples (Fig. 1B). The expression of MTOR in muscle fibers $(p<.0001)$, fibroblasts $(p<.01)$, macrophages $(p<.0001)$, and smooth muscle cells $(p<.05)$ were significantly different from muscle biopsy-extracted tissues. However, there was no significant difference in expression between the biopsied tissue cells, whole blood cells, endothelial cells, and muscle cells (Fig. 1C). Furthermore, MAPILC3A expression significantly differed among biopsied tissues and smooth muscle cells. However, MAP1LC3A expression in all other cell types, differed significantly from that in biopsied tissues (Fig. 1D). On the other hand y MAP1LC3B expression levels were similar in muscle fibers, macrophages, smooth muscle cells, biopsied tissues. However, whole blood cells, endothelial cells, fibroblasts, and muscle cells had significantly different MAP1LC $3 B$ expression compared to cells of the biopsied tissues ( $p<.0001$; Fig. 1E). Moreover, PRKAA1 expression in muscle fibers and macrophages was not significantly different from that in biopsied tissues. However, expression in whole blood cells, endothelial cells, fibroblasts, muscle cells, and smooth muscle cells differed significantly compared to that in biopsied tissue cells (Fig. 1F).

Notably, expression levels of AKT1, SLC2A4, MAPK1, and COX4I1 in muscle fibers, whole blood cells, endothelial cells, fibroblasts, macrophages, muscle cells, and smooth muscle cells differed significantly from those in biopsied muscle tissues ( $p<.0001$; Fig. 2A-D). Expression of MAPK14 in muscle fibers $(p<.01)$, whole blood cells $(p<.0001)$, and fibroblasts $(p<.001)$ was significantly different from that in biopsied tissues. However, MAPK14 expression did not differ significantly among endothelial cells, macrophages, muscle cells, smooth muscle cells, and biopsied tissues (Fig. 2E). Additionally, we determined that $M E F 2 A$ expression in macrophages and muscle cells was not significantly different from that in biopsied tissue cells. On the contrary, muscle fibers, whole blood cells, endothelial cells, fibroblasts, and smooth muscle cells exhibited significantly different expression of $M E F 2 A$ compared with cells from biopsied tissues ( $p<.001$; Fig. 2F). We observed that the difference in MAPK8 expression was insignificant among muscle fibers, endothelial cells, fibroblasts, and biopsied tissue cells. However, whole blood cells $(p<.0001)$, macrophages $(p<.0001)$, muscle cells $(p<.05)$, and smooth muscle cells $(p<.0001)$ expressed MAPK8 at significantly different levels compared with cells obtained from biopsied tissues (Fig. 2G). Moreover, RPS6KB1 expression in whole blood cells and endothelial cells did not differ significantly from that in biopsied tissues. On the other hand, $R P$ S6KB1 in muscle fibers $(p<.05)$, fibroblasts $(p<.001)$, macrophages $(p<.0001)$, muscle cells $(p<.0001)$, and smooth muscle cells $(p<.001)$ differed significantly from that in biopsied tissue samples (Fig. $2 \mathrm{H}$ ). Eventually, we observed that difference in SOD1 expression between smooth muscle cells and biopsied tissue cells was insignificant. However, SOD1 expression in muscle fibers, whole blood, endothelial cells, fibroblasts, macrophages, and muscle cells was significantly different from that in biopsied tissues ( $p<.0001$; Fig. 2I).

Kyung-Wan Baek, et al. • Gene Expression Analysis of the Skeletal Muscle Reveals 

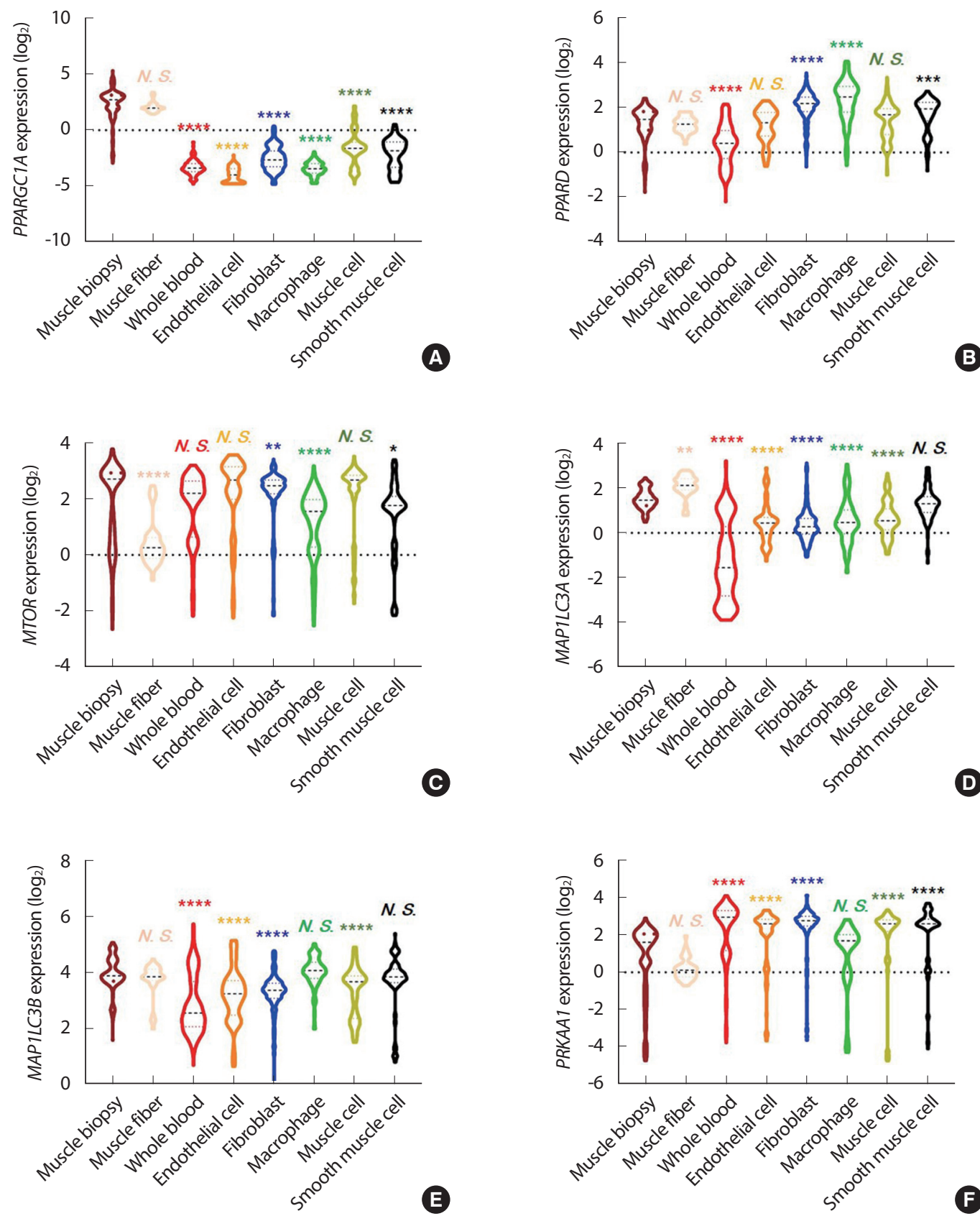

Fig. 1. Differences in expression of various genes analyzed in skeletal muscle according to cell types included in skeletal muscle among publication in the "Exercise Science" journal (Part 1). All data presented as the mean \pm standard deviation; vs. muscle biopsy. ${ }^{*} p<.05,{ }^{* *} p<.01,{ }^{* * *} p<.001$, and ${ }^{* * * *} p<.0001$. N. $\mathrm{S}=$ no significant difference compared to muscle biopsy.

\section{DISCUSSION}

Since physical activity is the movement of skeletal muscles [9], gene or protein expression that are altered in response to physical activity or exercise is a major concern in modern exercise science. Gene and protein expression regulated by physical exercise are experimentally validated by molecular biology techniques such as polymerase chain reaction or western blotting post animal-based experiments [10]. In addition, integrating microarray or RNA-sequencing data obtained from human tissues with meta-analysis can help gain insights on the effect of exercise in several diseases [11]. Thus, this can possibly explain the molecular mechanisms underlying the application of exercise as a treatment intervention for sarcopenia and muscle-related diseases in humans [12-14]. Moreover, it can also help in explaining skeletal muscles role as a primary secretory 

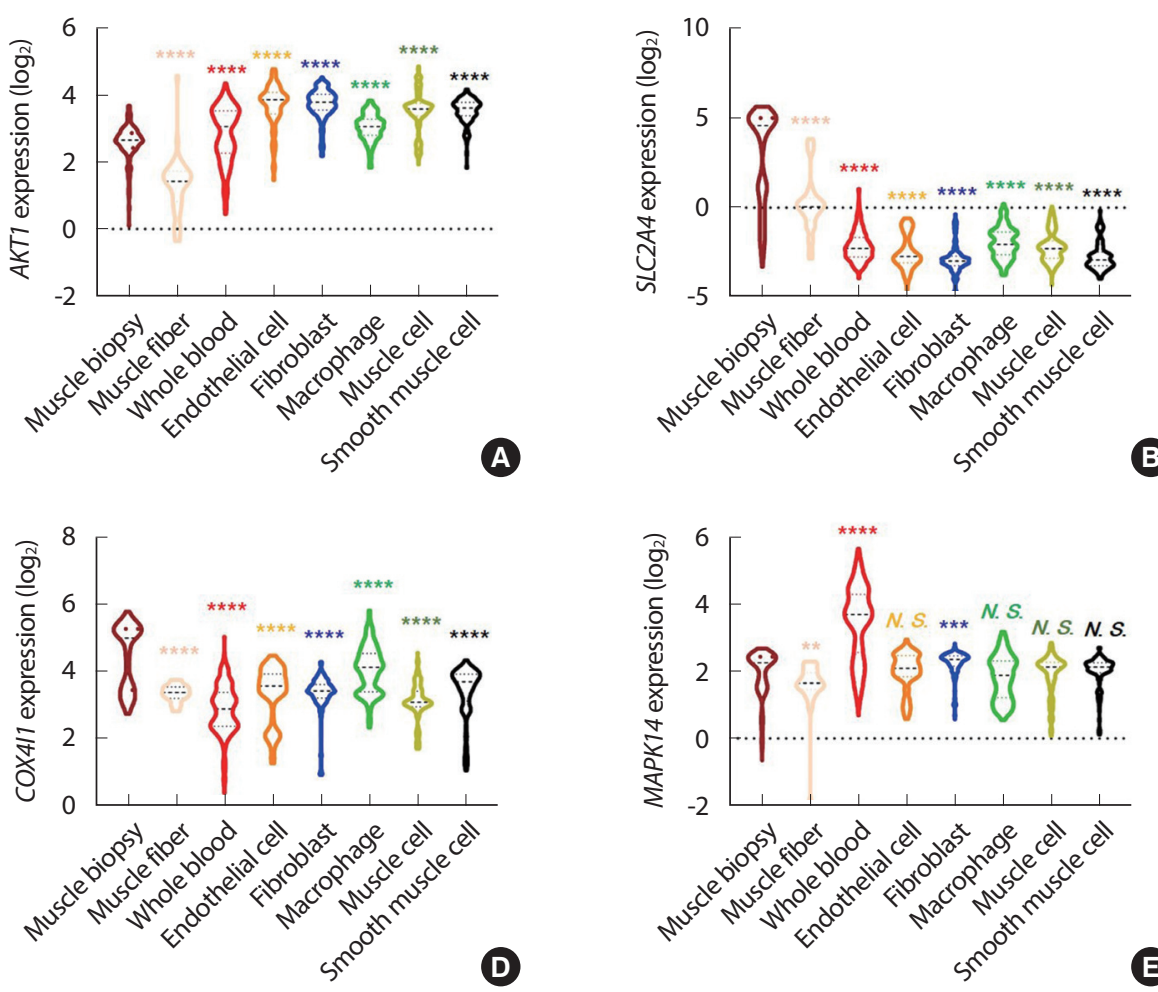

E

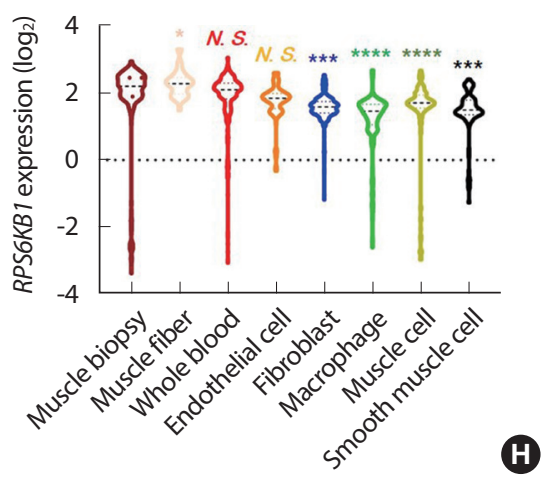

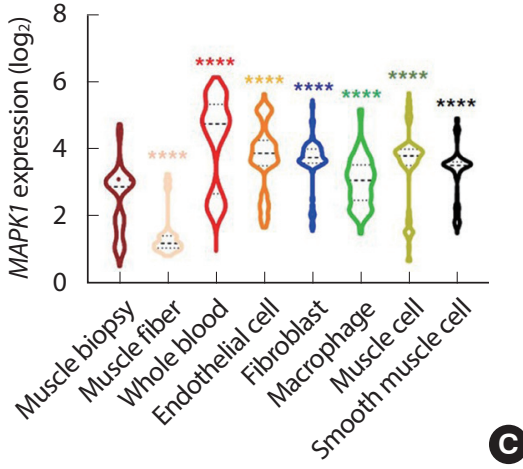

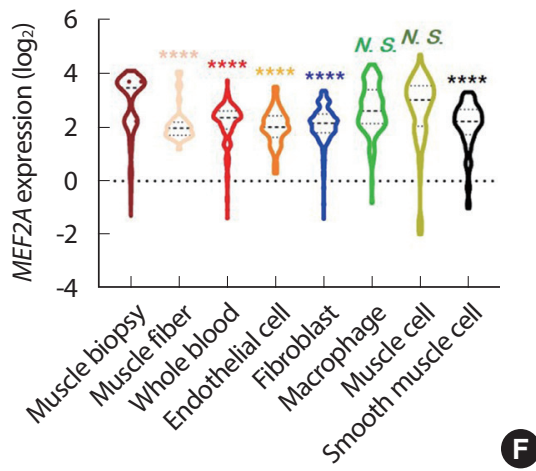

F

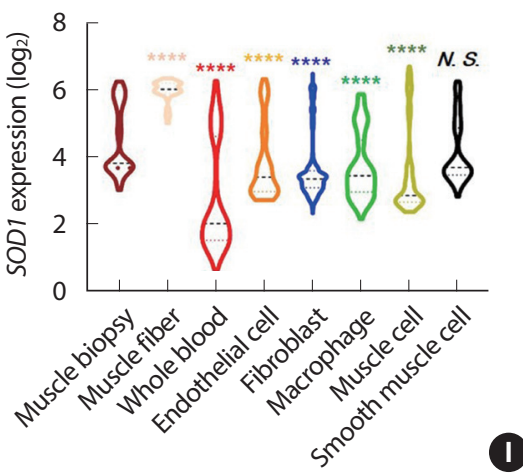

Fig. 2. Differences in expression of various genes analyzed in skeletal muscle according to cell types included in skeletal muscle among publication in the "Exercise Science" journal (Part 2). All data presented as the mean \pm standard deviation; vs. muscle biopsy. ${ }^{*} p<.05,{ }^{* *} p<.01,{ }^{* * *} p<.001$, and ${ }^{* * * *} p<.0001$. N. $\mathrm{S}=$ no significant difference compared to muscle biopsy.

organ in metabolic disorders [15]. Nevertheless, previous studies have failed to block differential gene and protein expression in cells as intervening factors in the skeletal muscle. Therefore, we conducted this study to prevent any misinterpretations of the expression of genes mainly analyzed in the field of exercise science. We aimed for our data to be helpful in future studies.

Exercise affects gene expression in almost every cell type that makes up the human body [16]. In addition, the effect on gene expression may differ depending on the type of exercise and the intensity of exercise or cell type [17]. Therefore, in order to determine the effect of exercise on skeletal muscle gene expression, it is necessary to conduct research by distinguishing the cell types constituting skeletal muscle. For example, hepatokines such as fetuin A may be released from the liver due to exercise [18], and the related gene expression may not be specific to skeletal muscle, it is because skeletal muscle contains blood. From a similar point of view, exercise may induce differences in macrophage polarization in some adipocytes in skeletal muscle in both acute and chronic exercise $[19,20]$. Therefore, based on various literature, finding the basis for whether there may be a difference in gene expression depending on the cell type and clarifying the target cell type will be the basis for the re- 
search plan. At the same time, if exercise scientists predict the difference according to cell type at the in silico and then conduct the research, it is thought that it will help the progress of the study to analyze the change in gene expression in skeletal muscle due to exercise.

Notably, we found that the expression of PPARGC1A, which is the most analyzed gene in "Exercise Science", did not differ significantly between biopsied muscle tissues and muscle fibers. However, we observed that it was differentially expressed in other cell types. This indicated that PPARGC1A is specifically expressed in skeletal muscle fibers. In addition, the significant difference in expression of AKT1, SLC2A4, MAPK1, and COX4I1 between biopsied cells and other cell types, indicated that the biopsied muscle tissue could be analyzed without additional pretreatment. Therefore, we determined that PPARGC1A, AKT1, SLC2A4, MAPK1, and COX4I1 are genes specifically expressed in the skeletal muscle. PGC-la (encoded by the PPARGC1A) is a transcriptional coactivator that regulates genes involved in energy metabolism (the master regulator of mitochondrial biogenesis) [21-23]. It also appears to be of major interest in exercise physiology, as endurance exercise is well known to activate PPARGC1A in rodent and human skeletal muscle [24]. Several publications in the "Exercise Science" journal also appear to reflect the characteristics of PPARGC1A, which may be involved in muscle metabolism, increase in skeletal muscle due to obesity, blood pressure regulation, cellular cholesterol homeostasis regulation, and obesity [25].

Barring the aforementioned genes, various genes may have differential gene expression in other cell types of the skeletal muscle. Therefore, depending on the purpose of the study, perfusion before animal sacrifice or conducting a pre-treatment (e.g., isolation) of the tissue after biopsy may be required. For example, perfusion may be a suitable method to evaluate skeletal muscle-specific gene expression of MTOR and RPS6KB1 that did not exhibit a significant difference in expression in whole blood cells. In addition, all other genes, including MTOR and RPS6KB1 but excluding skeletal muscle-specific genes, had no significant differences in their expression levels among one or more cell types. Therefore, an appropriate cell isolation process may be required depending on the purpose of the study.

\section{CONCLUSION}

We analyzed skeletal muscle-specific gene expression of genes most analyzed in the "Exercise Science" journal in the following order: $P P A R G$ C1A, PPARD, MTOR, MAPL1LC3A, MAPL1LC3B, PRKAA1, AKT1,

SLC2A4, MAPK1, COX4I1, MAPK14, MEF2A, MAPK8, and RPS6KB1. We verified that PPARGC1A, AKT1, SLC2A4, MAPK1, and COX4I1 were specifically expressed in muscle fibers or biopsied muscle tissues. However, other genes were found to have mixed gene expression levels in other cell types.

\section{ACKNOWLEDGEMENT}

Special thanks to Professor. Nicolas J. Pillon for his kind permission to use his online tools and database.

\section{CONFLICT OF INTEREST}

The authors declare that they do not have conflict of interest.

\section{AUTHOR CONTRIBUTIONS}

Conceptualization: KW Baek, SO Kwon; Data curation: KW Baek, S Kim; Formal analysis: KW Baek; Funding acquisition: SO Kwon; Methodology: KW Baek, SJ Kim; Project administration: KW Baek, SO Kwon; Visualization: KW Baek; Writing-original draft: KW Baek; Writing-review \& editing: KW Baek, JS Kim, SJ Kim, SO Kwon.

\section{ORCID}

Kyung-Wan Baek

So-Jeong Kim

Ji-Seok Kim

Sun-Ok Kwon

\section{REFERENCES}

1. Pilegaard H, Ordway GA, Saltin B, Neufer PD. Transcriptional regulation of gene expression in human skeletal muscle during recovery from exercise. Am J Physiol Endocrinol Metab. 2000;279(4):E806-14.

2. Tunstall RJ, Mehan KA, Wadley GD, Collier GR, Bonen A, et al. Exercise training increases lipid metabolism gene expression in human skeletal muscle. Am J Physiol Endocrinol Metab. 2002;283(1):E66-72.

3. Chan KL, Boroumand P, Milanski M, Pillon NJ, Bilan PJ, et al. Deconstructing metabolic inflammation using cellular systems. Am J Physiol Endocrinol Metab. 2017;312(4):E339-47. 
4. Pillon NJ, Krook A. Innate immune receptors in skeletal muscle metabolism. Exp Cell Res. 2017;360(1):47-54.

5. Jakubzick CV, Randolph GJ, Henson PM. Monocyte differentiation and antigen-presenting functions. Nat Rev Immunol. 2017;17(6):34962.

6. NCBI, Illumina bodyMap2 transcriptome. 2013; Retrived from: https://www.ncbi.nlm.nih.gov/bioproject/PRJEB2445/.

7. NCBI. MYH2 myosin heavy chain 2 [Homo sapiens (human)]. 2021; Retrieved from: https://www.ncbi.nlm.nih.gov/gene/4620.

8. Pillon NJ. Muscle Atlas. 2017; Retrieved from: https://nicopillon.com/ tools/muscle-atlas/.

9. Caspersen CJ, Powell KE, Christenson GM. Physical activity, exercise, and physical fitness: definitions and distinctions for health-related research. Public Health Rep. 1985;100(2):126-31.

10. Baek KW, Gim JA, Park JJ. Regular moderate aerobic exercise improves high-fat diet-induced nonalcoholic fatty liver disease via monoacylglycerol O-acyltransferase 1 pathway suppression. J Sport Health Sci. 2020;9(5):472-8.

11. Kang YJ, Yoo JI, Baek KW. Differential gene expression profile by RNA sequencing study of elderly osteoporotic hip fracture patients with sarcopenia. J Orthop Translat. 2021;29:10-8.

12. Baek KW, Kim JS, Yoo JI. Relationship between METTL21C gene expression and exercise in human skeletal muscle: a meta-analysis. Exerc Sci. 2021;30(1):102-9.

13. Baek KW, Jung YK, Kim JS, Park JS, Hah YS, et al. Rodent model of muscular atrophy for sarcopenia study. J Bone Metab. 2020;27(2):97110

14. Baek KW, Jung YK, Park JS, Kim JS, Hah YS, et al. Two types of mouse models for sarcopenia research: senescence acceleration and genetic modification models. J Bone Metab. 2021;28(3):179-91.

15. Baek KW, Lee DI, Jeong MJ, Kang SA, Choe Y, et al. Effects of lifelong spontaneous exercise on the M1/M2 macrophage polarization ratio and gene expression in adipose tissue of super-aged mice. Exp Gerontol. 2020;141:111091.

16. Fuller OK, Whitham M, Mathivanan S, Febbraio MA. The protective effect of exercise in neurodegenerative diseases: the potential role of extracellular vesicles. Cells. 2020;9(10):2182.

17. Ntanasis-Stathopoulos J, Tzanninis JG, Philippou A, Koutsilieris M. Epigenetic regulation on gene expression induced by physical exercise. J Musculoskelet Neuronal Interact. 2013;13(2):133-46.

18. Lee DI, Baek KW. A aingle bout of exercise inhibits alpha-2-HS-glyco- protein (fetuin A) in the adipose tissue of obese mice. Exercise Science. 2019;28(1):31-40.

19. Baek KW. Effects of a single of bout exercise on the macrophage phenotypic ratio in the adipose tissue of high-fat diet-induced obese mice. Exercise Science. 2018;27(3):232-43.

20. Baek KW, Lee DI, Kang SA, Yu HS. Differences in macrophage polarization in the adipose tissue of obese mice under various levels of exercise intensity. J Physiol Biochem. 2020;76(1):159-68.

21. Valero T. Mitochondrial biogenesis: pharmacological approaches. Curr Pharm Des. 2014;20(35):5507-9.

22. Dorn GW, 2nd, Vega RB, Kelly DP. Mitochondrial biogenesis and dynamics in the developing and diseased heart. Genes Dev. 2015;29(19): 1981-91.

23. Sanchis-Gomar F, Garcia-Gimenez JL, Gomez-Cabrera MC, Pallardo FV. Mitochondrial biogenesis in health and disease. Molecular and therapeutic approaches. Curr Pharm Des. 2014;20(35):5619-33.

24. Pilegaard H, Saltin B, Neufer PD. Exercise induces transient transcriptional activation of the PGC-1alpha gene in human skeletal muscle. J Physiol. 2003;546(Pt 3):851-8.

25. NCBI. PPARGC1A PPARG coactivator 1 alpha [Homo sapiens (human)]. 2021; Retrieved from: https://www.ncbi.nlm.nih.gov/gene?Db= gene\&Cmd=ShowDetailView\&TermToSearch $=10891$.

26. Lee DW, Oh S-H, Choi KO, Ju JS. The effects of 8-week acetic acid feeding on endurance performance and fat metabolism in skeletal muscle of mice. Exerc Sci. 2021;30(1):34-42.

27. Lim CH, Kim CK. Effect of acute low-load high-repetition resistance exercise on protein synthetic signaling pathway and satellite cell activation in skeletal muscle of rats. Exerc Sci. 2020;29(1):77-85.

28. Ahn N. Effects of combine exercise on HSP70 and SOD1 expression of aorta, skeletal muscle and myocardium in high fat diet induced obese aging rats. Exerc Sci. 2020;29(3):248-55.

29. Lim CH, Son HJ. Treadmill exercise increases muscle satellite cell activation independent of oxygen concentration in rat. Exerc Sci. 2019;28 (1):22-30

30. Oh SH, Lee DW, Lee YH, Ju JS. Resistance training ameliorates finasteride-induced disturbance in protein homeostasis in skeletal muscle of rats. Exerc Sci. 2019;28(2):159-67.

31. Chang YU, Kim JS. The effect of uphill and downhill exercise training on myogenic mechanism of Wnt/BMP signaling in the skeletal muscle of aged rats. Exerc Sci. 2019;28(2):182-90.

32. Lee JH, Zhang D, Kwak SE, Shin HE, Moon HY, et al. Effects of acute 
aerobic exercise on the early stage of energy metabolism related gene expression in mouse gastrocnemius: microarray analysis. Exerc Sci. 2019;28(1):49-59.

33. Namgoong H, Lee JS, Kim JG, Lee S. Acute effects of aerobic treadmill exercise intensity on expression of irisin and FNDC5 in male mouse. Exerc Sci. 2018;27(3):209-16.

34. Oh SL. BeadArray analysis of rat skeletal muscle to identify candidate genes of resistance exercise performance. Exerc Sci. 2016;25(1):10-7.

35. Hong YS, Oh SH, Lee DW, Ju JS. Autophagy flux Is decreased in response to endurance exercise training in aged mouse skeletal muscle. Exerc Sci. 2016;25(1):50-9.

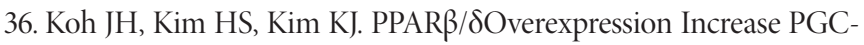
laProtein through the decreased PGC-1a ubiquitination in mouse skeletal muscle. Exerc Sci. 2015;24(4):407-14.

37. Kim SB, Kim JO, Yoon JH, Kim DS, Lee SH. Effects of aerobic exercise on PGC-1a, AMPK and SOD expression of skeletal muscle in 2Type diabetic rats. Exerc Sci. 2015;24(3):297-303.
38. Jung SR, Kim KJ, Koh JH. PGC-1a concentration in skeletal muscle by exercise is controlled by $\operatorname{PPAR} \beta / \delta$ through post-transcriptional mechanism: the effects of PPAR $\beta / \delta$ silence on PGC- $1 \alpha$ ubiquitination and stability. Exerc Sci. 2015;24(3):289-95.

39. Koh JH, Kim KJ, Jung SR, Kim SH. The increased PPAR $\beta / \delta$ by endurance exercise control MEF2A-GLUT4 and mitochodrial enzymes through NRF-1 promoter activity in mouse skeletal muscle. Exerc Sci. 2015;24(2):153-60.

40. Koh JH, Kim HS, Ahn N, Kim KJ. PPAR $\delta$ activation or overexpression attenuates PGC-1 1 protein degradation. Exerc Sci. 2015;24(1):4958.

41. Yoon SY, Kim SB, Yoon JH. The effects of combined treatment of chlorella intake and treadmill exercise training on MAP kinase activity in rat's skeletal muscle. Exerc Sci. 2015;24(1):67-74.

42. Kim SH, Kim KJ, Jung SR, Koh JH. Control of GLUT4 biogenesis through PPAR $\delta$ and MEF2 axis with single bout of endurance exercise in rodent skeletal muscle. Exerc Sci. 2015;24(1):59-66. 